\title{
Re-Emergent Tremor without Accompanying Rest Tremor in Parkinson's Disease
}

\author{
Elan D. Louis, Seth L. Pullman, David Eidelberg, Vijay Dhawan
}

Can. J. Neurol. Sci. 2008; 35: 513-515

A hallmark of Parkinson's disease (PD) is rest tremor, ${ }^{1}$ yet a variety of action tremors also occur in these patients. ${ }^{1-3}$ These action tremors are important because they are common and because motor disability in parkinsonism seems to correlate more with action than with rest tremor, ${ }^{1}$ making such tremors an under-appreciated target for treatment. Furthermore, PD patients with action tremor are commonly misdiagnosed as having essential tremor (ET), ${ }^{4,5}$ further adding to the importance of recognizing the variety of tremor phenomenologies seen in PD.

Re-emergent tremor is a postural tremor that emerges after a variable latency of several $(1-47$, mean $=9.4)$ seconds and has a frequency typical of Parkinsonian resting tremor $(4-6 \mathrm{~Hz}) .{ }^{1} \mathrm{It}$ is thought to represent a re-emergence of Parkinsonian rest tremor. We report a patient with PD who had no tremor at rest yet whose main complaint was a disabling re-emergent tremor. To our knowledge, this has not been reported previously and it therefore further expands the spectrum of motor phenomenology that clinicians can encounter in their patients with PD.

\section{Case Report}

At age 57 years, the patient first experienced clinical depression, although he had had a prior history of episodic mood disturbance. The initial treatment, lithium, was discontinued after one year. At age 58, he also developed features of parkinsonism, for which he was placed on levodopa-carbidopa $(25 / 1001$ tab bid).

He was initially seen at our center at age 61 years; his chief complaint was "severe shaking" of his right arm. There were no other complaints (e.g., no postural hypotension or impotence). There were no prior neurological problems and family history was unremarkable for neurological problems. His son has bipolar disorder and an aunt, depression (type unknown). His psychiatric medications were bupropion XL $300 \mathrm{mg} /$ day(d), lamotrigine 200 $\mathrm{mg} / \mathrm{d}$, valproate $1000 \mathrm{mg} / \mathrm{d}$, and gabapentin $1,800 \mathrm{mg} / \mathrm{d}$. Other medications included diltiazem LA $120 \mathrm{mg} / \mathrm{d}$. He had not taken any carbidopa-levodopa for two months. On examination, there were no orthostatic changes in blood pressure or heart rate. He was alert and fully oriented. Cranial nerves, strength and sensation were normal. Deep tendon reflexes were symmetrically diminished in all limbs with flexor plantar responses. No hypomimia was appreciated. There was no tremor at rest while lying down, seated, standing or walking during the 1.5 hour assessment. During sustained arm extension, a right arm tremor emerged after a latency of 1-7 seconds $($ mean $=2.2$ seconds). After emerging, the tremor worsened over the ensuing several seconds before reaching a plateau. Suggestibility, distraction, and tremor entrainment were all absent. The tremor primarily produced wrist flexion-extension movement but there was also an occasional rotatory component (with wrist pronation-supination) and occasional rhythmic thumb flexion. The right arm tremor emerged during several activities as well (finger-to-nose maneuver, writing). There was no tremor on the left during sustained arm extension and minimal tremor with movement (e.g., writing, finger-to-nose maneuver). Rapid alternating movements of both arms were notable for moderate bradykinesia with occasional pauses, and this was slightly worse on the right. Foot taps were mildly to moderately slow symmetrically bilaterally. His posture was slightly flexed and arm swing was bilaterally mildly-to-moderately decreased. With a moderate pull, he maintained balance. Rigidity, while absent on this initial examination, was present in both arms without reinforcement on all subsequent follow-up examinations (right greater than left), thereby demonstrating the presence of three hallmark features of PD (bradykinesia, rigidity, and tremor). Work up for hyperthyroidism and Wilson's disease was unremarkable. Brain MRI with and without contrast demonstrated mild age-related atrophy and minimal periventricular white matter changes. He was diagnosed with PD. (18)F-fluorodeoxyglucose positron emission tomography (FDG PET) revealed bilaterally increased uptake in the lentiform nucleus and thalamus, marginally more pronounced on the left, consistent with idiopathic PD. 6,7

The patient has received follow-up care for five years (30minute visits every three months). Rest tremor has not been observed, yet the re-emergent tremor has remained a source of disability; as a result, the patient performs many of his daily

\footnotetext{
From the GH Sergievsky Center (EDL), Department of Neurology (EDL, SLP), and Taub Institute for Research on Alzheimer's Disease and the Aging Brain (EDL), College of Physicians and Surgeons, Department of Epidemiology (EDL), Mailman School of Public Health, Columbia University, New York; Center for Neurosciences (DE, VD), The Feinstein Institute for Medical Research, North Shore-Long Island Jewish Health System, Manhasset, NY; Departments of Neurology and Medicine (DE, VD), New York University School of Medicine, New York, NY, USA.

Received February 14, 2008. Final Revisions Submitted March 31, 2008. Correspondence to: Elan Louis, Unit 198, Neurological Institute, 710 West 168th Street, New York, NY, 10032, USA.
} 
activities (writing, drinking, holding utensils, brushing his teeth) with his left hand. Low dose levodopa in combination with carbidopa noticeably improved his bradykinesia, but even at doses of $1750 \mathrm{mg} / \mathrm{d}$, did not provide any therapeutic benefit for his re-emergent tremor and resulted in cognitive side effects. Amantadine $(200 \mathrm{mg} / \mathrm{d})$ provided no tremor benefit and the tremor is not lessened with ethanol. The patient has also taken pramipexole ( $3 \mathrm{mg} /$ day), which resulted in mild improvement in rapid alternating movements and rigidity, but no improvement in tremor. He has also taken propranolol (20 mg/day), but there was no improvement in tremor.

His most recent neurological examination at age 66 years was similar to previous examinations, with the exception of moderate hypomimia and more (i.e., moderate) rigidity, which was present in all limbs. The re-emergent tremor was more severe than it was on his initial evaluation. His valproate and diltiazem had been discontinued for 1.5 years.

The patient underwent computerized tremor analysis and computerized spiral analysis in the Motor Physiology Laboratory (Columbia University). Experimental conditions have been described. ${ }^{8}$ Clinical conditions included: arms at rest, arms extended, finger-to-nose movements, writing and transferring water. Rest measurements were performed with the subject's arms flexed at 90 degrees, fully supported against gravity, and kept stationary at the elbow to prevent transmitted upper arm movements to the forearm and hand. Electromyelogram (EMG) confirmed that the tested limb was fully at rest. Spiral data were used to calculate several indices that quantified upper limb kinematics of spiral drawing in detail: (1) anisotropy (the relative dominance of tremor in a specific direction, e.g. if a tremor's direction was distributed over many angles, anisotropy is low, which is characteristic of parkinsonism), and (2) spiral speed (cm/second[s]) (slow speed, which may be evident in patients with PD, is a kinematic equivalent of parkinsonian bradykinesia). ${ }^{8}$

Tremor analysis did not reveal any tremors at rest. There were $5.6-6.5 \mathrm{~Hz}$ postural and kinetic tremors (severe on right and minimal on left) with alternating antagonist contractions and entrainment in the recorded EMG muscles at the same frequency on the right only. The postural tremor on the right emerged after several seconds (Figure). Inertial loading had minimal effect on tremor frequencies or amplitudes. Spiral analysis revealed severe right (average \pm standard deviation $(\mathrm{SD})$ power $=69.949 \pm$ $46.712 \mathrm{~mm}^{2}$ ) and minimal left (power $\left.=0.112 \pm 0.039 \mathrm{~mm}^{2}\right) 4.8$ - 6.0 Hz kinetic tremors. There was also a severely decreased spiral speed on the left $(1.169 \pm 0.436 \mathrm{~cm} / \mathrm{s}$ vs. laboratory normal $=8.821 \pm 2.831 \mathrm{~cm} / \mathrm{s})$ and decreased anisotropy $(0.864 \pm 0.144$ on the left and $0.916 \pm 0.052$ on the right). These findings are all characteristic of action parkinsonism.

\section{DisCuSSION}

Our patient's tremor had characteristics of re-emergent tremor, including its latency ( 2 - 4 seconds in our patient and 1 47 seconds in previous reports) ${ }^{1}$ and relatively slow frequency. The tremor continued during movement, which is a feature that has been described in patients with re-emergent tremor. One issue is whether the tremor should be referred to as a "reemergent" tremor, as there is no accompanying tremor at rest. In

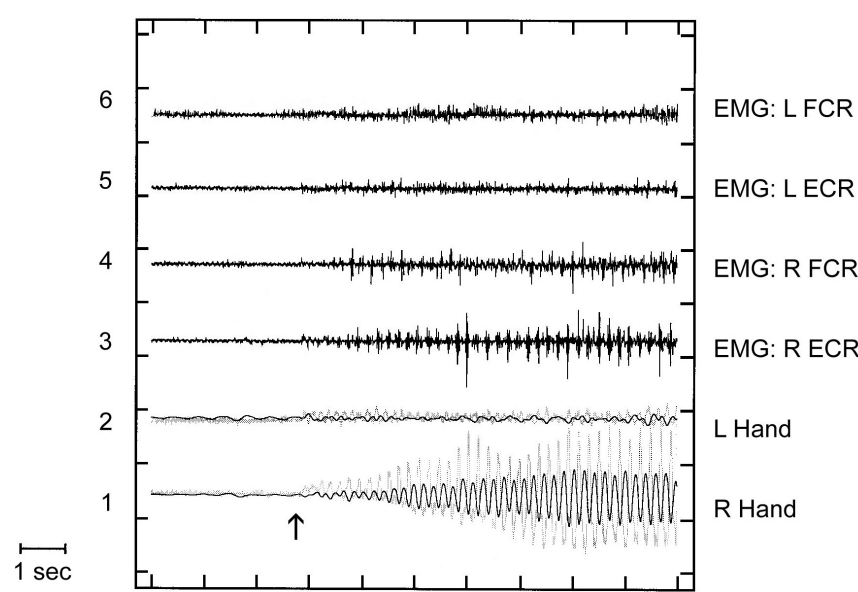

Figure: Tracing from the patient with his arms extended at the shoulders over a 10 second epoch. The bottom two channels are accelerometric (light lines) and displacement (dark lines) recordings from the right and left hands. Channels 3 - 6 are right $(R)$ and $(L)$ surface EMG recordings from the flexor carpi radialis (FCR) and extensor carpi radialis (ECR). These data illustrate the onset of re-emergent tremor, evident in the right hand, beginning approximately 3 seconds into the tracings at the arrow. Note onset from virtually no tremor to a crescendo-ing, then stabilizing, tremor in the right hand with associated increasing EMG activity. On the left, there is a minimal, tonic increase in EMG activity; on the right, alternating antagonist bursts of EMG discharges drive the re-emergent tremor.

the absence of a tremor at rest, it might be appropriate to refer to the tremor we observed as an "emergent" tremor.

The patient was evaluated in detail at age 61 years and then on a regular basis (four times per year over a five year period). Rest tremor has never been observed while seated, standing, lying, or walking. Similarly, subtle rest tremor was not observed during tremor analysis.

It is unlikely that our patient's postural tremor was merely the result of ET on top of parkinsonism (two diagnoses). The tremor emerged after a latency of several $(2-4)$ seconds; by contrast, in ET, the tremor usually occurs as soon as the limb assumes a horizontal posture (i.e., latency $=0$ seconds). ${ }^{1}$ The frequency $(4.8-6.8 \mathrm{~Hz})$ was typical of parkinsonian resting tremor (approximately $4-6 \mathrm{~Hz}$ ) and similar to that reported by Jankovic et $\mathrm{al}^{1}$ for re-emergent tremor $(5.5 \mathrm{~Hz})$. The tremor was characterized by occasional wrist pronation-supination and occasional rhythmic thumb flexion, which is more typical of the tremor of parkinsonism than of ET. ${ }^{9}$ Furthermore, the reemergent tremor in our patient was unilateral. Unilateral tremor is a typical feature of Parkinsonian tremor and rarely of ET. ${ }^{4}$ The lack of responsiveness to ethanol, absence of a family history of tremor and absence of head or voice tremor also argue against ET.

Our patient had underlying PD as evidenced by bradykinesia, rigidity (not present in initial examination but present in all examinations since then), clear response of bradykinesia to low dose levodopa therapy, and supporting quantitative physiologic 
findings and FDG PET imaging results. ${ }^{6,7}$ The FDG-PET was used instead of FDOPA in order to not only confirm PD but also to rule out the presence of an atypical parkinsonian variant. ${ }^{10}$

Although bradykinesia and rigidity were more marked on the right, they were present bilaterally. By contrast, re-emergent tremor was present only on the right. Why was this one feature unilateral while the other features were not? We have previously noted in a clinical sample of 179 PD cases, that there was no association between the rest tremor score and bradykinesia and rigidity scores. ${ }^{11}$ There is also additional evidence that the tremor in PD may represent a different underlying pathophysiological process than the rigidity and bradykinesia. First, factor analysis of signs in PD showed that rest tremor was relatively independent of the other cardinal signs of PD. ${ }^{12}$ Second, we previously reported that while bradykinesia and rigidity worsened at similar annual rates in PD, rest tremor did not clearly worsen with time. ${ }^{13}$ Finally, there was no correlation between rest tremor and striatal 18F-dopa uptake in PD patients. ${ }^{14}$

One issue is that during several of his examinations, he had been taking either valproate or diltiazem, and each of these medications rarely can result in drug-induced parkinsonism. ${ }^{15,16}$ We do not think that this tremor was drug-induced for the following reasons: First, the PET scan results indicated unequivocal bilateral lentiform glucose hypermetabolism, a finding that is consistent with idiopathic PD. ${ }^{6,7}$ Second, aside from tremor, the patient has other hallmark features of PD, including bradykinesia and rigidity, and these have persisted despite being off of these drugs for 1.5 years. Third, we are unaware of a report in the literature of a drug producing this clinical picture (i.e., a unilateral re-emergent tremor without rest tremor). Finally, the re-emergent tremor has worsened over the past five years, which is consistent with an underlying progressive degenerative condition like PD.

The tremor was unusual and the patient had a psychiatric history; hence, a functional movement disorder was also considered. However, the consistency of findings (including the consistent tremor frequency), and the absence of suggestibility, distraction, and tremor entrainment on examination argued against this possibility, as did the PET imaging results.

Patients with PD can exhibit a variety of types of tremor other than the classic rest tremor. The presence of other tremor types, particularly when they occur in the absence of rest tremor, can result in diagnostic confusion and diagnostic errors. The presence of a severe action tremor, in particular, is a common reason for mis-assigning a diagnosis of ET to patients who actually have PD, with as many as $15 \%$ of such "ET" patients actually having mis-diagnosed PD. ${ }^{3}$ Such diagnostic errors can result in the institution of the wrong set of pharmacotherapeutic interventions and delays in the institution of neuroprotective therapies.

\section{Conclusions}

In summary, we clinically and electrophysiologically characterized a patient with PD who had severe re-emergent tremor yet no rest tremor. These observations serve to further expand the spectrum of the tremor phenomenology that confronts physicians who encounter patients with PD.

\section{ACKNOWLEDGEMENTS}

Supported by R01 NSO42859 from the National Institutes of Health (Bethesda, MD).

\section{REFERENCES}

1. Jankovic J, Schwartz KS, Ondo W. Re-emergent tremor of Parkinson's disease. J Neuro Neurosurg Psychiatry. 1999;67: 646-50.

2. Lance JW, Schwab RS, Peterson EA. Action tremor and the cogwheel phenomenon in Parkinson's disease. Brain. 1963;86: 95-110.

3. Zesiewicz TA, Hauser RA. Phenomenology and treatment of tremor disorders. Neurol Clin. 2001;19:651-80

4. Jain S, Lo S, Louis E. Essential tremor may be the most commonly mis-diagnosed movement disorder: identification of factors associated with diagnostic errors. Neurology. 2006;66:A114.

5. Benito-Leon J, Louis ED. Essential tremor: emerging views of a common disorder. Nat Clin Pract Neurol. 2006;2:666-78.

6. Fukuda M, Edwards C, Eidelberg D. Functional brain networks in Parkinson's disease. Parkinsonism Relat Disord. 2001;8:91-4.

7. Eckert T, Barnes A, Dhawan V, Frucht S, Gordon MF, Feigin AS, et al. FDG PET in the differential diagnosis of parkinsonian disorders. Neuroimage. 2005;26:912-21.

8. Cohen O, Pullman S, Jurewicz E, Watner D, Louis ED. Rest tremor in essential tremor patients: prevalence, clinical correlates, and electrophysiological characteristics. Arch Neurol. 2003;60: 405-10.

9. Louis ED. Essential tremor. Clin Geriatr Med. 2006;22:843-57.

10. Eckert G, Barnes A, Dhawan V, Frucht S, Gordon MF, Feigin AS, et al. FDG PET in the differential diagnosis of parkinsonian disorders. Neuroimage. 2005;26:912-21.

11. Louis ED, Levy G, Cote LJ, Mejia H, Fahn S, Marder K. Clinical correlates of action tremor in Parkinson's disease. Arch Neurol. 2001;58:1630-4

12. Zetusky WJ, Jankovic J, Pirozzolo FJ. The heterogeneity of Parkinson's disease: clinical and prognostic implications. Neurology. 1985;35:522-6.

13. Louis ED, Tang MX, Cote L, Alfaro B, Mejia H, Marder K. Progression of extrapyramidal signs in Parkinson's disease. Arch Neurol. 1999;56:334-7.

14. Vingerhoets FJ, Schulzer M, Calne DB, Snow BJ. Which clinical sign of Parkinson's disease best reflects the nigrostriatal lesion? Ann Neurol. 1997;41:58-64.

15. Graham DF, Stewart-Wynne EG. Diltiazem-induced acute parkinsonism. Aust NZ J Med. 1994;24:70.

16. Esterford K, Clough P, Kellett M, Fallon K, Duncan S. Reversible parkinsonism with normal B-CIT-SPECT in patients exposed to sodium valproate. Neurology. 2004;62:1435-7. 